\title{
Orbitofrontal sulcogyral pattern and olfactory sulcus depth in the schizophrenia spectrum
}

Yumiko Nishikawa ${ }^{1,{ }^{*}}$, Tsutomu Takahashi ${ }^{1}$, Yoichiro Takayanagi ${ }^{1}$, Atsushi Furuichi ${ }^{1}$, Mikio Kido ${ }^{1}$, Mihoko Nakamura ${ }^{1}$, Daiki Sasabayashi ${ }^{1}$, Kyo Noguchi ${ }^{2}$, Michio Suzuki ${ }^{1}$

1. Department of Neuropsychiatry, University of Toyama Graduate School of Medicine and Pharmaceutical Sciences, 2630 Sugitani, Toyama 930-0194, Japan

2. Department of Radiology, University of Toyama Graduate School of Medicine and Pharmaceutical Sciences, 2630 Sugitani, Toyama 930-0194, Japan

*Corresponding author: Yumiko Nishikawa, M.D.

Department of Neuropsychiatry, University of Toyama Graduate School of Medicine and Pharmaceutical Sciences, 2630 Sugitani, Toyama 930-0194, Japan

Tel: +81-76-434-2281

Fax: +81-76-434-5030

E-mail: yumiko@med.u-toyama.ac.jp 


\begin{abstract}
Morphological changes in the orbitofrontal cortex (OFC), such as an altered sulcogyral pattern of the 'H-shaped' orbital sulcus and a shallow olfactory sulcus, have been demonstrated in schizophrenia, possibly reflecting deviations in early neurodevelopment. However, it remains unclear whether patients with schizotypal features exhibit similar OFC changes. This magnetic resonance imaging (MRI) study examined the OFC sulcogyral pattern (Types I, II, III, and IV) and olfactory sulcus morphology in 102 patients with schizophrenia, 47 patients with schizotypal disorder, and 84 healthy controls. The OFC sulcogyral pattern distribution between the groups was significantly different on the right hemisphere, with the schizophrenia patients showing a decrease in Type I ( $v s$ controls and schizotypal patients) and an increase in Type III ( $v s$ controls) expression. However, the schizotypal patients and controls did not differ in the OFC pattern. There were significant group differences in the olfactory sulcus depth bilaterally (schizophrenia patients $<$ schizotypal patients $<$ controls). Our findings suggest that schizotypal disorder, a milder form of schizophrenia spectrum disorders, partly shares the OFC changes (i.e., altered depth of the olfactory sulcus) with schizophrenia, possibly reflecting a common disease vulnerability. However, altered distribution of the OFC pattern specific to schizophrenia may at least partly reflect neurodevelopmental pathology related to a greater susceptibility to overt psychosis.
\end{abstract}

\title{
Keywords
}

Orbitofrontal cortex $\cdot$ Olfactory sulcus $\cdot$ Magnetic resonance imaging $\cdot$ Schizophrenia $\bullet$ Schizotypal disorder 


\section{Introduction}

Schizotypal (personality) disorder (SPD) is thought to be a prototypic disorder within the schizophrenia spectrum $[1,2]$, characterized by odd behavior and attenuated forms of schizophrenic features without the manifestation of an overt and sustained psychosis $[3,4]$. SPD is genetically related to schizophrenia and might share biological and psychological commonalities with schizophrenia as a neurobiological basis for vulnerability factors $[2,5,6]$. A previous magnetic resonance imaging (MRI) study demonstrated malformation of the adhesio interthalamica, a midline brain structure related to neurodevelopment during early gestation [7], in both schizophrenia and schizotypal patients, possibly reflecting common neurodevelopmental deviations [8]. However, it remains largely unknown whether the timing and degree of the neurodevelopmental pathology differs between overt schizophrenia and the milder form of schizophrenia spectrum disorder.

Several MRI studies of schizophrenia have investigated variations in the orbitofrontal cortex (OFC) 'H-shaped' sulcus [Type I, II, and III] [9] and demonstrated increased Type III and decreased Type I expression on the right hemisphere in schizophrenia $[10,11,12]$. Given that the OFC H-shaped sulcus develops predominantly during the mid (around 28 weeks) to late gestation period and remains rather stable after birth $[13,14,15]$, these findings suggest that genetic and/or environmental events in neurodevelopment during such gestation period may be related to the pathophysiology of schizophrenia. The depth of the olfactory sulcus, which appears in the fetal forebrain at around 16 weeks gestation [14], is another possible neurodevelopmental marker located on the OFC. Although not consistently replicated [16], a shallow olfactory sulcus has been reported in early $[17,18]$ as well as chronic [19] phases of schizophrenia, possibly reflecting deviant forebrain development during early gestation. These potential neurodevelopmental markers originating from different 
developmental periods may offer a clue regarding the estimation of the gestational age at which neurodevelopmental events occur in the schizophrenia spectrum. To our knowledge, however, no MRI studies have investigated the OFC sulcogyral pattern and/or olfactory sulcus depth in subjects with schizotypal features.

The present study aimed to investigate both the OFC patterns and olfactory sulcus morphology in schizophrenia patients, schizotypal disorder patients, and healthy control subjects. On the basis of the concept of the schizophrenia spectrum [2] as well as previous MRI findings [8], we predicted that schizophrenia and schizotypal patients would exhibit similar but partly different gross brain changes, which might be more deviant from normal in schizophrenia than in schizotypal disorder. We also investigated the association between these potential neurodevelopmental markers and clinical features (e.g., symptom severity, medication) in schizophrenia spectrum disorders.

\section{Materials and Methods}

Subjects

Forty-seven schizotypal disorder patients, 102 schizophrenia patients, and 84 control subjects were included in this study. The subjects were right-handed except for one female patient with schizotypal disorder who was of unknown handedness. Table 1 shows the demographic and clinical data of the subjects.

The schizotypal disorder patients who met the ICD-10 criteria for research [20] were recruited from among patients who visited the clinics of the Department of Neuropsychiatry, Toyama University Hospital. This patient group exhibited schizotypal features accompanied by distress or associated problems in their lives and required clinical care including low-dose antipsychotics. The sample characteristics of the clinic-based schizotypal disorder patients in our laboratory have been described 
previously $[21,22,23,24]$. The mental condition of each subject was assessed by experienced psychiatrists to check for the emergence of overt psychotic symptoms, and none of the 47 patients has developed overt schizophrenia to date (mean follow-up period after MRI scanning $=3.0$ years, $\mathrm{SD}=2.6$ ). All available clinical information and data obtained from a detailed review of the clinical records and structured interviews for the Comprehensive Assessment of Symptoms and History (CASH) [25], including the chapter on premorbid or intermorbid personality, were stored in a database. The subjects were diagnosed by consensus reached by at least two psychiatrists based on these data. Although all of the schizotypal subjects in this study also fulfilled the DSM-IV criteria of the SPD on Axis II, 13 subjects had previously experienced transient quasi-psychotic episodes fulfilling a DSM Axis I diagnosis of brief psychotic disorder [3]. At the time of the MRI scanning, 40 of the 47 patients were treated with low-dose antipsychotics. Of those 40 patients, 14 were treated with typical antipsychotics and 26 received atypical antipsychotics. The remaining seven patients were neuroleptic-naïve.

The schizophrenia patients fulfilled the ICD-10 diagnostic criteria for schizophrenia [20]. All but two of the schizophrenia patients were on antipsychotic medication; 42 were being treated with typical antipsychotics, 57 were receiving atypical antipsychotics, and one was receiving both typical and atypical. The clinical symptoms of the schizotypal disorder and the schizophrenia patients were rated at the time of scanning using the Scale for the Assessment of Negative Symptoms (SANS) and the Scale for the Assessment of Positive Symptoms (SAPS) [26, 27].

The control subjects of similar age range as the patient groups were recruited from among members of the community, hospital staff, and university students. They were given a questionnaire consisting of 15 items concerning their personal (13 items; e.g. a history of obstetric complications, substantial head injury, seizures, neurological or psychiatric diseases, impaired thyroid function, hypertension, diabetes, and substance 
use) and family (two items) histories of illness.

All subjects were physically healthy at the time of the study, and none had a lifetime history of serious head trauma, neurological illness or substance abuse disorder. All participants were also screened for gross brain abnormalities by the neuroradiologists. The olfactory sulcus depth of 64/102 schizophrenia patients and 64/84 controls in this study has been reported elsewhere [17]. Forty-five schizophrenia patients and 37 controls in this study were also used to investigate the possible role of genetic variation on the OFC patterns [28]. There was no sample overlap between this study and Takayanagi et al. [12], where we demonstrated altered OFC patterns in first-episode schizophrenia. This study was approved by the Committee on Medical Ethics of Toyama University. After a complete description of the study, written informed consent was obtained from all subjects.

Magnetic resonance imaging procedures

The magnetic resonance images were obtained by utilizing a 1.5-T Magnetom Vision (Siemens Medical System, Inc, Erlangen, Germany) with a three-dimensional gradient-echo sequence FLASH (fast low-angle shots) yielding 160-180 contiguous T1-weighted slices of 1.0-mm thickness in the sagittal plane. The imaging parameters were: repetition time $=24 \mathrm{~ms}$; echo time $=5 \mathrm{~ms}$; flip angle $=40^{\circ}$; field of view $=256$ $\mathrm{mm}$; and matrix size $=256 \times 256$ pixels. The voxel size was $1.0 \times 1.0 \times 1.0 \mathrm{~mm}$.

The image data were processed on a Linux PC (Fujitsu, Tokyo, Japan) using the software package Dr View (AJS, Tokyo, Japan). Brain images were realigned in three dimensions to standardize for differences in head tilt during image acquisition and were then reconstructed into entire contiguous coronal images, with a 1-mm thickness, perpendicular to the anterior commissure-posterior commissure line. The intracranial volume (ICV) was measured to correct for differences in head size as described 
previously [29]; there was no group difference in the ICV (Table 3).

OFC sulcogyral pattern classification

The medial orbital sulcus (MOS), lateral orbital sulcus (LOS), and transverse orbital sulcus (TOS) were highlighted on consecutive 1-mm coronal slices, and then viewed in the axial plane for OFC pattern classification based on the definition by Chiavaras and Petrides [9]. Briefly, the OFC sulcogyral patterns were classified according to the continuity of the 'H-shaped' sulcus consisting of the MOS, TOS, and LOS; for Type I the MOS is disconnected while the LOS is intact, for Type II both the MOS and LOS are continuous, and for Type III both the MOS and LOS are disconnected (Fig. 1. Also, see Bartholomeusz et al. [30]). In rare instances where the MOS was continuous, but the LOS was disconnected, this pattern was classified as Type IV [10]. Two raters (YN and TT), who were blind to the subjects' identity, independently performed the OFC sulcogyral pattern classification for all subjects. Intra- (YN) and inter-rater reliabilities (Cronbach's $\alpha$ ) in a subset of 20 randomly selected brains (40 hemispheres) were over 0.81. A consensus agreement was reached in all cases even when the initial classification differed between the raters.

Olfactory sulcus measurements

As described in detail elsewhere [17], one rater (TT), who was blind to the subjects' identity, measured the depth of the olfactory sulcus in all 1-mm coronal slices where the sulcus could be identified (Fig. 2). On each coronal slice, the olfactory sulcus was traced beginning with the deepest point of the sulcus and ending inferiorly with a tangent line connecting the top surfaces of the gyrus rectus and medial orbital gyrus [31]. The length of the sulcus in the anterior-posterior direction ( $\mathrm{mm}$ ) was equal to the 
number of these coronal slices. The average depth of the sulcus on each hemisphere was calculated as follows: sum of the depth in all slices containing the sulcus / slice number [i.e., sulcus length (mm)]. Intra- and inter-rater (TT and YN) intraclass correlation coefficients for the sulcus depth in 10 randomly selected brains were over 0.83 .

Statistical analysis

Clinical and demographic differences between groups were examined with a one-way analysis of variance (ANOVA) or $\chi^{2}$ test. Group differences in the OFC sulcogyral pattern distribution were evaluated using the $\chi^{2}$ test. The relationships between the sulcogyral pattern and the clinical/demographic variables were analyzed for each hemisphere using ANOVA with the OFC sulcogyral pattern (Type I-III) as a between-subject factor. The hemispheres with the Type IV pattern $(N=3)$ were excluded from the statistical analyses. The average depth and length of the olfactory sulcus were analyzed using the repeated measures analysis of covariance (ANCOVA), with age and ICV as covariates (these factors did not relate to each other; Supplementary material 1), diagnosis and gender as between-subject factors, and hemisphere as a within-subject variable. Age was not normally distributed (Kolmogorov-Smirnov test, $p<0.001$ ), but the findings of this study did not change even when we used log-transformed age in the statistical analyses. The olfactory sulcus depth in the plane of the posterior tangent through the eyeballs (PPTE) (Fig. 2) was also analyzed using the same model in order to test if single-slice approach could replicate the results of entire sulcus measurements. The schizophrenia group was also divided into first-episode $(N=65$, defined as illness duration $\leq 1$ year or under first psychiatric hospitalization [17], age $=24.0 \pm 4.6$ years, illness duration $=1.0 \pm 1.0$ years) and chronic $(N=37$, age $=28.2 \pm 5.7$ years, illness duration $=8.2 \pm 4.9$ years $)$ subgroups in order to examine possible effects of illness chronicity and/or medication. The 
relationship between the olfactory sulcus depth and the clinical variables was examined by Pearson's partial correlation coefficients controlling for ICV. In order to examine the relationship between the OFC pattern and olfactory sulcus depth for each hemisphere, the olfactory sulcus depth was analyzed by ANCOVAs with age and ICV as covariates, and diagnosis and OFC pattern as between-subject factors. Post-hoc Scheffé's tests were used to follow-up these ANOVAs/ANCOVAs. Statistical significance was defined as $p<0.05$ (two-tailed).

\section{Results}

Demographic and clinical data

The three groups did not differ significantly in age, gender ratio, height, and parental education. As predicted, the control subjects had attained a higher mean level of education than had the patients with either disorder (Table 1). The total SAPS score for the schizophrenia patients was significantly higher than that for the schizotypal patients. There were significant differences in medication dosage and duration of antipsychotic medication; the patients with schizotypal disorder took significantly smaller amounts of antipsychotics than did the patients with schizophrenia.

Group difference in the OFC pattern distribution

The OFC sulcogyral patterns were significantly different between the groups in the right hemisphere (Table 2, Supplementary material 2), with the schizophrenia patients having increased Type III ( $v$ s controls, $\chi^{2}=8.37, p=0.004$; $v$ s schizotypal, $\chi^{2}=5.32, p=$ 0.021 ) and decreased Type I ( $v s$ controls, $\chi^{2}=5.62, p=0.018 ; v s$ schizotypal, $\chi^{2}=3.57$, $p=0.059$ ) expression. However, there was no difference in the right OFC pattern 
between the schizotypal and control subjects $\left(\chi^{2}<0.01, p=0.998\right)$. Gender did not significantly affect the OFC sulcogyral pattern (left, $\chi^{2}=0.105, p=0.950$; right, $\chi^{2}=$ 2.41, $p=0.301)$.

OFC pattern and clinical/demographic variables

In the schizophrenia and schizotypal patients as a whole, the patients with right type II had a significantly lower total SAPS score $[F(2,137)=4.63, p=0.011]$ compared with those with type I $(p=0.011)$ or type III $(p=0.033)$ patterns. There were no significant effects of the OFC pattern on demographic (age, education, and parental education) or other clinical (onset age, illness duration, medication, and SANS scores in the patients) variables.

Olfactory sulcus measures

ANCOVA of the olfactory sulcus length showed no significant effect involving diagnosis (Table 3). However, that for depth revealed significant main effects of diagnosis $[F(2,225)=99.79, p<0.001]$ and hemisphere $[F(1,227)=86.08, p<$ $0.001]$ but no interaction between these factors $[F(2,227)=1.04, p=0.356]$. Post-hoc analyses showed that the depth of this sulcus became increasingly more superficial as one moved from controls to schizotypal patients to schizophrenia patients (all $p<0.001$, Table 3). The sulcus depth was significantly deeper in the right than in the left hemisphere for all diagnostic groups $(p<0.001)$. These findings did not change even when we used gender as a covariate, but not as a between-subject factor, in the ANCOVA. The olfactory sulcus depth did not correlate with age in either group (Supplementary material 1). In the patient groups, sulcus depth did not significantly correlate with clinical variables [onset age and illness duration for schizophrenia 
patients and medication (dose, duration) and total SANS/SAPS scores for both disorders].

The sulcus depth in the PPTE slice significantly correlated with the average depth in all diagnostic groups for both hemispheres $(r=0.371$ to 0.593 , all $p<0.010)$. However, group comparisons using the PPTE slice failed to detect the difference between schizotypal and schizophrenia patients (post-hoc test, $p=0.087$ ).

Relation between the OFC pattern and olfactory sulcus depth

The ANCOVAs demonstrated no significant effect of the OFC pattern or OFC pattern-by-diagnosis interaction on the olfactory sulcus depth for both left and right hemispheres.

Possible effect of illness stages on the orbitofrontal changes

The OFC pattern did not differ between the first-episode and chronic schizophrenia patients for both left $\left(\chi^{2}=0.79, p=0.674\right)$ and right $\left(\chi^{2}=3.53, p=0.171\right)$ hemispheres. The first-episode patients had increased Type III ( $v s$ controls, $\chi^{2}=11.81, p<0.001$; vs schizotypal, $\left.\chi^{2}=7.95, p=0.005\right)$ and decreased Type I ( $v s$ controls, $\chi^{2}=8.76, p=$ 0.003 ; vs schizotypal, $\chi^{2}=5.99, p=0.014$ ) expression on the right hemisphere. However, we did not find significant difference in the OFC sulcogyral pattern distribution between the chronic schizophrenia and controls or schizotypal patients probably due to reduced statistical power.

There was no difference in the olfactory sulcus depth between the first-episode and chronic schizophrenia patients $(p=0.987)$. In addition, both first-episode ( $v s$ controls, $p$ $<0.001 ; v s$ schizotypal, $p=0.003$ ) and chronic ( $v s$ controls, $p<0.001 ; v s$ schizotypal, $p$ $=0.034)$ schizophrenia subgroups had significantly shallower sulcus as compared with 
controls and schizotypal patients."

\section{Discussion}

To our knowledge, this is the first MRI study to report both the OFC sulcogyral pattern and olfactory sulcus depth in the schizophrenia spectrum disorders. The OFC pattern distribution was significantly different between the groups on the right hemisphere; patients with schizophrenia, but not those with schizotypal disorder, had a decrease in Type I and an increase in Type III expression as compared with healthy comparisons. Both schizophrenia and schizotypal patients had a shallower olfactory sulcus compared to the controls, but its degree was significantly greater in schizophrenia. These findings suggest that greater morphologic deviations in potential neurodevelopmental markers may be at least partly related to overt and sustained psychosis observed in schizophrenia.

Consistent with previous MRI findings of the OFC sulcogyral pattern in independent cohorts $[10,11,12]$, the present study demonstrated increased Type III and decreased Type I expression on the right hemisphere in schizophrenia. Our controls, as well as those of Bartholomeusz et al. [30] (left Type II, 17.8\%; right Type II, 11.0\%), had a somewhat lower prevalence of Type II compared to previous reports. However, such a difference may be partly attributable to different sample characteristics as well as different OFC pattern classification methods between the studies; we and Bartholomeusz et al. [29] traced the main sulci on consecutive coronal slices and classified the OFC patterns also using axial and sagittal views, which could detect subtle disconnection of the MOS/LOS and tend to result in lower prevalence of Type II (i.e., both the MOS and LOS are continuous), whereas some other studies [11, 12] defined the OFC patterns predominantly by surface analyses in axial slices. Interestingly, recent studies have demonstrated an altered OFC pattern prior to the onset 
of psychosis $[10,32]$, supporting the idea that these OFC findings reflect neurodevelopmental deviations during the mid to late gestation period at which the OFC H-shaped sulcus develops $[13,14,15]$. On the other hand, we found no significant alteration of the OFC pattern in schizotypal disorder patients, implicating a less deviant neurodevelopmental change in the frontal region in this milder form of schizophrenia spectrum disorders. Previous neuroimaging studies in SPD have revealed preserved or even larger-than-normal prefrontal cortex volume, possibly reflecting protective factors against full-blown psychosis $[5,6,23]$. SPD is also characterized by attenuated frontal-striatal-temporal dysconnectivity compared with schizophrenia patients, which may mitigate the emergence of psychosis [33]. The present and those previous observations in the schizophrenia spectrum may be partly consistent with the hypothesis in schizophrenia that early neurodevelopmental deviations render the brain vulnerable to anomalous post-pubertal neurodevelopmental processes, as indicated by evidence for accelerated gray matter loss and aberrant connectivity particularly in prefrontal regions [34].

Another main finding of this study was a greater change of the olfactory sulcus depth, but not length, in schizophrenia than in schizotypal patients as compared with controls. In contrast to single-slice approach using external landmarks (i.e., eyeballs), which failed to detect olfactory sulcus changes in schizophrenia [16], we measured the entire olfactory sulcus in order to detect its subtle group difference. Since the olfactory sulcus appears during fetal development at around 16 weeks of gestation and is prominent at 25 weeks [14], in combination with previous findings of altered olfactory sulcus in various stages of schizophrenia $[17,18,19,35]$, the present results suggested deviant forebrain development during early gestation especially in schizophrenia. Nevertheless, although its degree was milder than in schizophrenia, schizotypal patients also exhibited a shallow olfactory sulcus, possibly reflecting common neurodevelopmental pathology within the schizophrenia spectrum. Our earlier results of 
midline brain structure in the schizophrenia spectrum [8] partly parallel these findings; we found abnormally small adhesio interthalamica that develops at around 13 to 14 weeks of gestation [7] in both schizophrenia and schizotypal disorder patients. Taken together with our findings in the OFC patterns, the patients with schizophrenia spectrum may partly share aberrant neurodevelopment early in gestation as a neurobiological basis for vulnerability factors, while greater and more prolonged deviations during the mid to late gestation period as well as consequent anomalous post-pubertal neurodevelopmental processes, especially in prefrontal regions, may lead to the overt and sustained psychosis observed in full-blown schizophrenia. Since discrepant findings, such as reduced prefrontal volume in SPD [36] or normal olfactory sulcus depth in schizophrenia [16], have also been reported, further comprehensive assessment of potential neurodevelopmental markers as well as the relation to cortical findings in the schizophrenia spectrum would be required to test our hypothesis.

Several limitations of the current study should be taken into account. First, most of the current schizophrenia and schizotypal patients were receiving antipsychotics at the time of scanning, whereas medication with antipsychotics could significantly affect brain morphology $[37,38,39]$. It is also possible that our unexpected finding of the relation between Type II OFC pattern and less severe psychotic symptoms in the schizophrenia spectrum might be due to the use of medicated samples. Although we found no significant effect of medication on the OFC pattern and olfactory sulcus depth, the present findings should be replicated using medication-naïve patients. Second, the present cross-sectional study cannot directly answer whether our findings reflect disease vulnerability (i.e., static neurodevelopmental risk marker). Gross cortical folding patterns are at least partly genetically mediated [28] and remain rather stable throughout life in healthy subjects $[13,40]$. However, the olfactory sulcus depth could be affected by active gray matter loss in the frontal cortex suggested as occurring during or after the onset of psychosis [41, 42, 43], which might reflect an increased rate of synaptic 
pruning and myelination $[44,45]$. Our previous longitudinal study revealed no progressive changes in the olfactory sulcus in first-episode schizophrenia [17], whereas illness chronicity might affect the sulcus depth later in the course of the illness [34]. Although we found no difference in the OFC morphology between the first-episode and chronic schizophrenia subgroups in this study, longitudinal MRI analysis would be required to investigate the stability of our findings in various illness stages in order to establish the role of the potential neurodevelopmental markers examined in this study. Finally, as the OFC pattern is also reported to be associated with autism spectrum disorders [46] and depressive psychopathology in young adolescents [47], the disease specificity of our findings should be tested in future studies.

In conclusion, our findings suggest that schizotypal disorder, a milder form of schizophrenia spectrum disorders, partly shares an altered depth of the olfactory sulcus with overt schizophrenia, possibly reflecting a common disease vulnerability. However, only the schizophrenia patients exhibited deviations in the OFC pattern distribution as compared with healthy controls. Our results, as well as the time point at which these potential neurodevelopmental markers develop during the gestation period, suggest that overt schizophrenia may be related to greater and/or more prolonged neurodevelopmental deviations. 


\section{Acknowledgements}

This research was supported in part by Grants-in-Aid for Scientific Research (C) (No. 22591275, 24591699, 26461739) and Grants-in-Aid for Scientific Research (B) (No. 24390281) from the Japanese Society for the Promotion of Science, Health and Labour Sciences Research Grants (Comprehensive Research on Disability, Health and Welfare, H23-Seishin-Ippan-002 and H23-Seishin-Ippan-009), and Research Grants from the JSPS Asian Core Program and the Research Group For Schizophrenia, Japan. The authors would like to thank Prof. Hideki Origasa (Department of Biostatistics and Clinical Epidemiology, University of Toyama Graduate School of Medicine and Pharmaceutical Sciences) for his support in statistical analyses. We would also like to thank the radiological technologists, especially Mr. Koichi Mori and Mr. Sadanori Ito, who assisted in the MRI data collection at Toyama University Hospital. 


\section{Conflict of Interest}

There are no conflicts of interest for any of the authors including any financial, personal or other relationships with other people or organizations within three years of beginning the submitted work that could inappropriately influence, or be perceived to influence, our work. 


\section{References}

1. Siever LJ, Kalus OF, Keefe RSE (1993) The boundaries of schizophrenia. Psychiatr Clin North Am 16:217-244

2. Siever LJ, Davis KL (2004) The pathophysiology of schizophrenia disorders: perspective from the spectrum. Am J Psychiatry 161, 398-413

3. American Psychiatric Association (1994) Diagnostic and Statistical Manual of Mental Disorders, 4th edn. American Psychiatric Association Press, Washington, $\mathrm{DC}$

4. World Health Organization (1992) The ICD-10 Classification of Mental and Behavioural Disorders: Clinical Descriptions and Diagnostic Guidelines. World Health Organization, Geneva

5. Fervaha G, Remington G (2013) Neuroimaging findings in schizotypal personality disorder: a systematic review. Prog Neuropsychopharmacol Biol Psychiatry 43:96-107

6. Hazlett EA, Goldstein KE, Kolaitis JC (2012) A review of structural MRI and diffusion tensor imaging in schizotypal personality disorder. Curr Psychiatry Rep $14: 70-78$

7. Rosales RK, Lemay MJ, Yakovley PI (1968) The development and involution of massa intermedia with regard to age and sex. J Neuropathol Exp Neurol 27:166

8. Takahashi T, Suzuki M, Zhou SY, Nakamura K, Tanino R, Kawasaki Y, Seal ML, Seto H, Kurachi M (2008) Prevalence and length of the adhesio interthalamica in schizophrenia spectrum disorders. Psychiatry Res 164:90-94

9. Chiavaras MM, Petrides M (2000) Orbitofrontal sulci of the human and macaque monkey brain. J Comp Neurol:422, 35-54

10. Chakirova G, Welch KA, Moorhead TW, Stanfield AC, Hall J, Skehel P, Brown VJ, Johnstone EC, Owens DG, Lawrie SM, McIntosh AM (2010) Orbitofrontal 
morphology in people at high risk of developing schizophrenia. Eur Psychiatry $25: 366-372$

11. Nakamura M, Nestor PG, McCarley RW, Levitt JJ, Hsu L, Kawashima T, Niznikiewicz M, Shenton ME (2007) Altered orbitofrontal sulcogyral pattern in schizophrenia. Brain 130:693-707

12. Takayanagi Y, Takahashi T, Orikabe L, Masuda N, Mozue Y, Nakamura K, Kawasaki Y, Itokawa M, Sato Y, Yamasue H, Kasai K, Okazaki Y, Suzuki M (2010) Volume reduction and altered sulco-gyral pattern of the orbitofrontal cortex in first-episode schizophrenia. Schizophr Res 121:55-65

13. Armstrong E, Schleicher A, Omran H, Curtis M, Zilles K (1995) The ontogeny of human gyrification. Cereb Cortex 5:56-63

14. Chi JG, Dooling EC, Gilles FH (1977) Gyral development of the human brain. Ann Neurol 1:86-93

15. Kringelbach ML, Rolls ET (2004) The functional neuroanatomy of the human orbitofrontal cortex: evidence from neuroimaging and neuropsychology. Prog Neurobiol 72:341-372

16. Nguyen AD, Pelavin PE, Shenton ME, Chilakamarri P, McCarley RW, Nestor PG, Levitt JJ (2011) Olfactory sulcal depth and olfactory bulb volume in patients with schizophrenia: an MRI study. Brain Imaging Behav 5:252-261

17. Takahashi T, Nakamura Y, Nakamura K, Ikeda E, Furuichi A, Kido M, Kawasaki Y, Noguchi K, Seto H, Suzuki M (2013) Altered depth of the olfactory sulcus in first-episode schizophrenia. Prog Neuropsychopharmacol Biol Psychiatry $40: 167-172$

18. Takahashi T, Nakamura Y, Nakamura K, Nishiyama S, Ikeda E, Furuichi A, Kido M, Noguchi K, Suzuki M (2013) Altered depth of the olfactory sulcus in subjects at risk of psychosis. Schizophr Res 149:186-187

19. Turetsky BI, Crutchley P, Walker J, Gur RE, Moberg PJ (2009) Depth of the 
olfactory sulcus: a marker of early embryonic disruption in schizophrenia?

Schizophr Res 115:8-11

20. World Health Organization (1993) The ICD-10 Classification of Mental and Behavioural Disorders: Diagnostic Criteria for Research. World Health Organization, Geneva

21. Kawasaki Y, Suzuki M, Nohara S, Hagino H, Matsui M, Yamashita I, Takahashi T, Chitnis X, McGuire PK, Seto H, Kurachi M (2004) Structural brain differences in patients with schizotypal disorder and schizophrenia demonstrated by voxel-based morphometry. Eur Arch Psychiatry Clin Neurosci 254:406-414

22. Suzuki M, Zhou SY, Hagino H, Takahashi T, Kawasaki Y, Nohara S, Yamashita I, Matsui M, Seto H, Kurachi M (2004) Volume reduction of the right anterior limb of the internal capsule in patients with schizotypal disorder. Psychiatry Res $130: 213-225$

23. Suzuki M, Zhou SY, Takahashi T, Hagino H, Kawasaki Y, Niu L, Matsui M, Seto H, Kurachi M (2005) Differential contributions of prefrontal and temporolimbic pathology to mechanisms of psychosis. Brain 128:2109-2122

24. Takahashi T, Suzuki M, Zhou SY, Tanino R, Hagino H, Kawasaki Y, Matsui M, Seto H, Kurachi M (2006) Morphologic alterations of the parcellated superior temporal gyrus in schizophrenia spectrum. Schizophr Res 83:131-143

25. Andreasen NC, Flaum M, Arndt S (1992) The Comprehensive Assessment of Symptoms and History (CASH): an instrument for assessing diagnosis and psychopathology. Arch Gen Psychiatry 49:615-623

26. Andreasen NC (1984) The Scale for the Assessment of Negative Symptoms (SANS). The University of Iowa, Iowa City, IA

27. Andreasen NC (1984) The Scale for the Assessment of Positive Symptoms (SAPS). The University of Iowa, Iowa City, IA

28. Takahashi T, Nakamura Y, Nakamura Y, Aleksic B, Takayanagi Y, Furuichi A, 
Kido M, Nakamura M, Sasabayashi D, Ikeda M, Noguchi K, Kaibuchi K, Iwata N, Ozaki N, Suzuki M (2014) The polymorphism of $Y W H A E$, a gene encoding 14-3-3epsilon, and orbitofrontal sulcogyral pattern in patients with schizophrenia and healthy subjects. Prog Neuropsychopharmacol Biol Psychiatry 51:166-171

29. Zhou SY, Suzuki M, Hagino H, Takahashi T, Kawasaki Y, Nohara S, Yamashita I, Seto H, Kurachi M (2003) Decreased volume and increased asymmetry of the anterior limb of the internal capsule in patients with schizophrenia. Biol Psychiatry 54: $427-436$

30. Bartholomeusz CF, Whittle SL, Montague A, Ansell B, McGorry PD, Velakoulis D, Pantelis C, Wood SJ (2013) Sulcogyral patterns and morphological abnormalities of the orbitofrontal cortex in psychosis. Prog Neuropsychopharmacol Biol Psychiatry 44:168-177

31. Rombaux P, Grandin C, Duprez T (2009) How to measure olfactory bulb volume and olfactory sulcus depth? B-ENT 5 Suppl 13:53-60

32. Lavoie S, Bartholomeuz CF, Nelson B, Lin A, McGorry PD, Velakoulis D, Whittle SL, Yung AR, Pantelis C, Wood SJ (2014) Sulcogyral pattern and sulcal count of the orbitofrontal cortex in individuals at ultra high risk for psychosis. Schizophr Res 154:93-99

33. Lener MS, Wong E, Tang CY, Byne W, Goldstein KE, Blair NJ, Haznedar MM, New AS, Chemerinski E, Chu KW, Rimsky LS, Siever LJ, Koenigsberg HW, Hazlett EA (2014) White matter abnormalities in schizophrenia and schizotypal personality disorder. Schizophr Bull, in press. doi: 10.1093/schbul/sbu093

34. Pantelis C, Yücel M, Wood SJ, Velakoulis D, Sun D, Berger G, Stuart GW, Yung A, Phillips L, McGorry PD (2005) Structural brain imaging evidence for multiple pathological processes at different stages of brain development in schizophrenia. Schizophr Bull 31:672-696

35. Takahashi T, Wood SJ, Yung AR, Nelson B, Lin A, Yücel M, Phillips LJ, 
Nakamura Y, Suzuki M, Brewer WJ, Proffitt TM, McGorry PD, Velakoulis D, Pantelis C (2014) Altered depth of the olfactory sulcus in ultra high-risk individuals and patients with psychotic disorders. Schizophr Res 153:18-24

36. Raine A, Lencz T, Yaralian P, Bihrle S, LaCasse L, Ventura J, Colletti P (2002) Prefrontal structural and functional deficits in schizotypal personality disorder. Schizophr Bull 28:501-513

37. Andreasen NC, Liu D, Ziebell S, Vora A, Ho BC (2013) Relapse duration, treatment intensity, and brain tissue loss in schizophrenia: a prospective longitudinal MRI study. Am J Psychiatry 170:609-615

38. Lieberman JA, Tollefson GD, Charles C, Zipursky R, Sharma T, Kahn RS, Keefe RS, Green AI, Gur RE, McEvoy J, Perkins D, Hamer RM, Gu H, Tohen M, HGDH Study Group (2005) Antipsychotic drug effects on brain morphology in first-episode psychosis. Arch Gen Psychiatry 62:361-370

39. Moncrieff J, Leo J (2010) A systematic review of the effects of antipsychotic drugs on brain volume. Psychol Med 40:1409-1422

40. Magnotta VA, Andreasen NC, Schultz SK, Harris G, Cizadlo T, Heckel D, Nopoulos P, Flaum M (1999) Quantitative in vivo measurement of gyrification in the human brain: changes associated with aging. Cereb Cortex 9:151-160

41. Pantelis C, Velakoulis D, Wood SJ, Yücel M, Yung AR, Phillips LJ, Sun DQ, McGorry PD (2007) Neuroimaging and emerging psychotic disorders: the Melbourne ultra-high risk studies. Int Rev Psychiatry 19:371-381

42. van Haren NE, Schnack HG, Cahn W, van den Heuvel MP, Lepage C, Collins L, Evans AC, Hulshoff Pol HE, Kahn RS (2011) Changes in cortical thickness during the course of illness in schizophrenia. Arch Gen Psychiatry 68:871-880

43. Sun D, Stuart GW, Jenkinson M, Wood SJ, McGorry PD, Velakoulis D, van Erp TG, Thompson PM, Toga AW, Smith DJ, Cannon TD, Pantelis C (2009) Brain surface contraction mapped in first-episode schizophrenia: a longitudinal magnetic 
resonance imaging study. Mol Psychiatry 14:976-86

44. Huttenlocher PR (1979) Synaptic density in human frontal cortex-developmental changes and effects of aging. Brain Res 163:195-205

45. Sowell ER, Peterson BS, Thompson PM, Welcome SE, Henkenius AL, Toga AW (2003) Mapping cortical change across the human life span. Nat Neurosci 6:309315

46. Watanabe H, Nakamura M, Ohno T, Itahashi T, Tanaka E, Ohta H, Yamada T, Kanai C, Iwanami A, Kato N, Hashimoto R (2014) Altered orbitofrontal sulcogyral patterns in adult males with high-functioning autism spectrum disorders. Soc Cogn Affect Neurosci 9:520-528

47. Whittle S, Bartholomeusz C, Yücel M, Dennison M, Vijayakumar N, Allen NB (2014) Orbitofrontal sulcogyral patterns are related to temperamental risk for psychopathology. Soc Cogn Affect Neurosci 9:232-239 


\section{Figure Legends}

Fig. 1

Classification of the orbitofrontal sulcogyral pattern on an axial view parallel to the anterior commissure-posterior commissure line. Note that these sulci were identified using orthogonal views in three directions and colored on consecutive coronal slices. c, caudal portion; LOS, lateral orbital sulcus; MOS, medial orbital sulcus; r, rostral portion; TOS, transverse orbital sulcus.

Fig. 2

Olfactory sulci on coronal (A) and axial (B) views, which were colored on 1-mm consecutive coronal slices (see panels $\mathrm{D}$ to $\mathrm{N}$ for sample coronal slices of the left olfactory sulcus). Panel A and the dotted line on panel B show the plane of the posterior tangent through the eyeballs (PPTE). Close-up coronal view of the left olfactory sulcus (C) shows the landmarks for the sulcus measurements [deepest point of the sulcus (arrow head) and a tangent line connecting the top surfaces of the gyrus rectus (GR) and medial orbital gyrus (MOG)]. As we traced the surface of the intrasulcal gray matter using a line of $1-\mathrm{mm}$ width, the number of pixels $(1.0 \times 1.0 \mathrm{~mm})$ in each coronal slice was equal to the sulcus depth (mm). 


\section{Supplementary materials}

\section{Supplementary material 1}

Scatter plots for the olfactory sulcus depth, age, and intracranial volume (ICV) in each diagnostic group. Only the age among these variables was not normally distributed (Kolmogorov-Smirnov test, $p<0.001$ ), but there was no quadratic (left, $p=0.34$; right, $p=0.37$ ) or cubic (left, $p=0.44$; right, $p=0.50$ ) correlation between age and sulcus depth.

Supplementary material 2

Distribution of the orbitofrontal sulcogyral pattern in each diagnostic group. ${ }^{*} p<0.01$, $* * p<0.05$ 
Type I

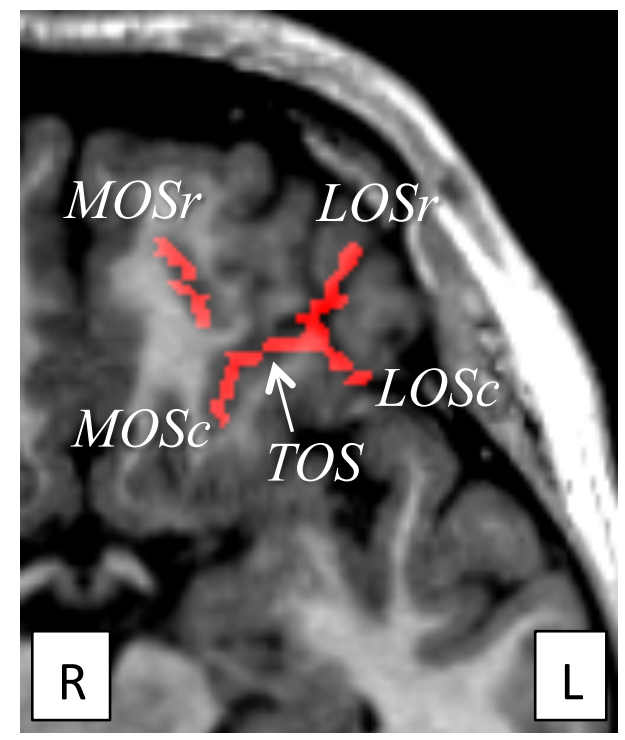

Type II

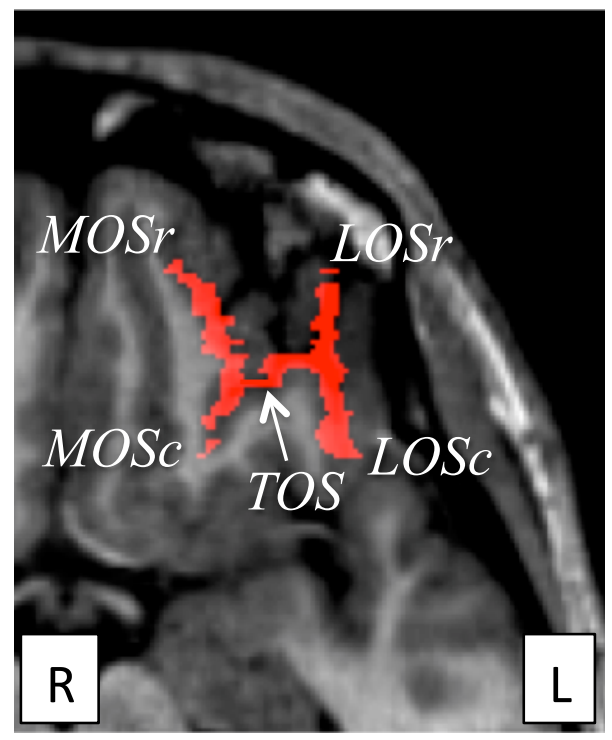

Type III

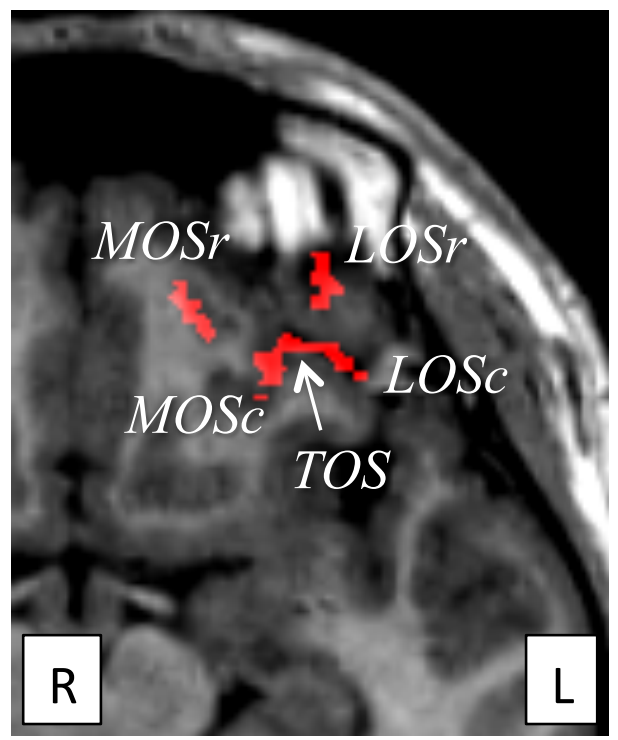

Type IV

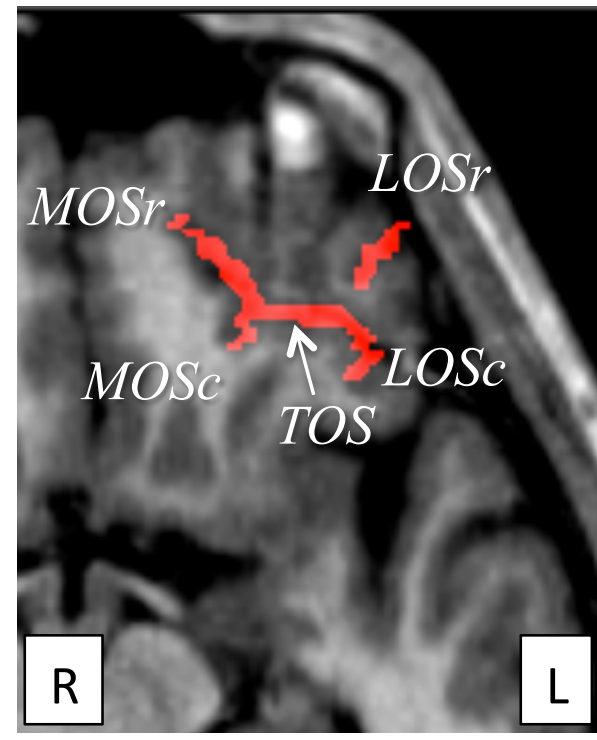




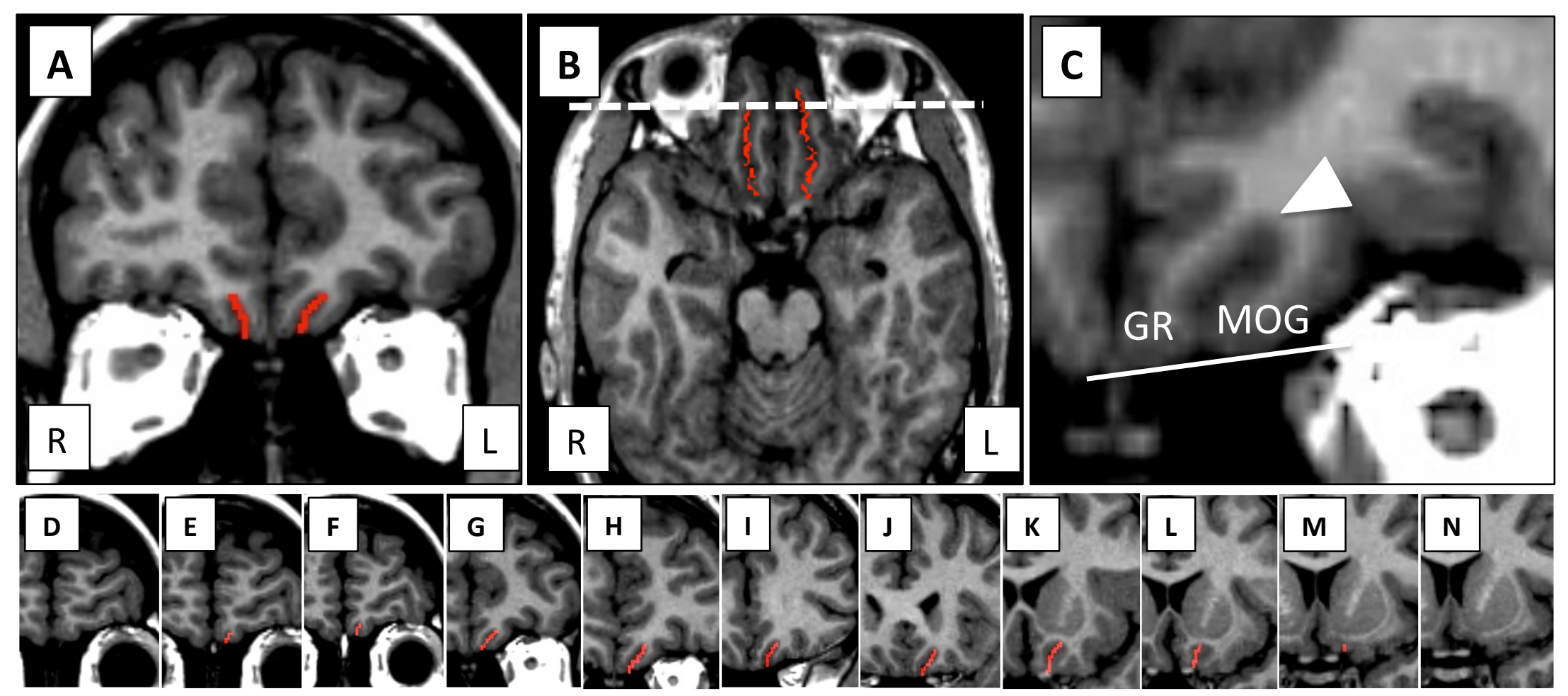


Table 1. Demographic and clinical data of healthy controls, patients with schizotypal disorder, and patients with schizophrenia

\begin{tabular}{|c|c|c|c|c|}
\hline & Controls & Schizotypal & Schizophrenia & Group comparisons \\
\hline Male/female & $47 / 37$ & $29 / 18$ & $55 / 47$ & $x^{2}=0.80, p=0.672$ \\
\hline Age (years) [range] & $24.5 \pm 5.7[18.0-45.9]$ & $25.0 \pm 5.4[16.0-37.0]$ & $25.5 \pm 5.5[16.7-45.6]$ & $F(2,230)=0.72, p=0.487$ \\
\hline Height $(\mathrm{cm})$ & $166.5 \pm 7.7$ & $165.9 \pm 8.7$ & $164.7 \pm 8.0$ & $F(2,230)=1.28, p=0.281$ \\
\hline Education (years) & $16.0 \pm 2.5$ & $13.1 \pm 2.0$ & $13.4 \pm 1.9$ & $F(2,230)=42.67, p<0.001 ;$ Con $>$ Sz, SzTypal \\
\hline Parental education (years) ${ }^{a}$ & $12.9 \pm 2.3$ & $12.3 \pm 1.7$ & $12.4 \pm 2.1$ & $F(2,221)=1.81, p=0.166$ \\
\hline Age at onset (years) & - & - & $22.0 \pm 4.5$ & - \\
\hline Duration of illness (years) & - & - & $3.6 \pm 4.6$ & - \\
\hline Duration of medication (years) & - & $1.5 \pm 3.0$ & $2.6 \pm 3.9$ & $F(1,147)=2.61, p=0.108$ \\
\hline Drug (mg/day, haloperidol equivalent) & - & $4.8 \pm 5.7$ & $10.3 \pm 8.9$ & $F(1,147)=15.30, p<0.001 ; \mathrm{Sz}>$ SzTypal \\
\hline Total SAPS score ${ }^{a}$ & - & $16.0 \pm 9.2$ & $27.8 \pm 21.3$ & $F(1,139)=12.65, p<0.001 ; \mathrm{Sz}>$ SzTypal \\
\hline Total SANS score $^{a}$ & - & $41.9 \pm 21.7$ & $49.1 \pm 23.4$ & $F(1,139)=3.10, p=0.081$ \\
\hline
\end{tabular}

Values represent means \pm SDs unless otherwise stated.

${ }^{a}$ Data missing for some participants.

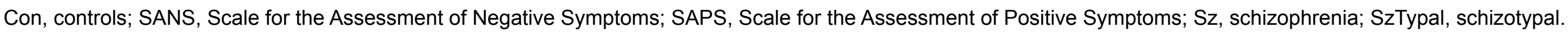


Table 2. Distribution of OFC sulcogyral pattern in the study participants

\begin{tabular}{|c|c|c|c|c|c|}
\hline & $\begin{array}{l}\text { Controls } \\
(N=84) \\
\end{array}$ & $\begin{array}{c}\text { Schizotypal } \\
(N=47) \\
\end{array}$ & $\begin{array}{c}\text { Schizophrenia } \\
\quad(N=102) \\
\end{array}$ & $x^{2}$ & $p$ \\
\hline & $N(\%)$ & $N(\%)$ & $N(\%)$ & & \\
\hline Left hemisphere & & & & 6.80 & 0.147 \\
\hline Type I & $50(59.5)$ & $27(57.5)$ & $51(50.0)$ & & \\
\hline Type II & $16(19.1)$ & $4(8.5)$ & $15(14.7)$ & & \\
\hline Type III & $17(20.2)$ & 15 (31.9) & $36(35.3)$ & & \\
\hline Type IV & $1(1.2)$ & $1(2.1)$ & $0(0.0)$ & & \\
\hline Right hemisphere & & & & 10.84 & 0.028 \\
\hline Type I & $63(75.0)$ & $35(74.5)$ & $59(57.8)$ & & \\
\hline Type II & $7(8.3)$ & $4(8.5)$ & $6(5.9)$ & & \\
\hline Type III & $14(16.7)$ & $8(17.0)$ & $36(35.3)$ & & \\
\hline Type IV & $0(0.0)$ & $0(0.0)$ & $1(1.0)$ & & \\
\hline
\end{tabular}


Table 3. Olfactory sulcus measures and intracranial volume in the study participants.

\begin{tabular}{|c|c|c|c|c|}
\hline & $\begin{array}{l}\text { Controls } \\
(N=84)\end{array}$ & $\begin{array}{l}\text { Schizotypal } \\
\qquad(N=47)\end{array}$ & $\begin{array}{l}\text { Schizophrenia } \\
\qquad(N=102)\end{array}$ & Diagnosis effect \\
\hline Olfactory sulcus depth (mm) & & & & $F(2,225)=99.79, p<0.001$ \\
\hline Left & $13.5 \pm 1.1^{a}$ & $12.2 \pm 1.1^{b}$ & $11.4 \pm 1.3$ & \\
\hline Right & $14.3 \pm 1.2^{\mathrm{C}}$ & $12.8 \pm 1.0^{\mathrm{C}}$ & $12.1 \pm 1.3^{\mathrm{C}}$ & \\
\hline Olfactory sulcus length (mm) & & & & $F(2,225)=0.15, p=0.858$ \\
\hline Left & $42.4 \pm 3.0$ & $41.9 \pm 2.6$ & $41.8 \pm 3.5$ & \\
\hline Right & $42.4 \pm 3.2$ & $42.7 \pm 2.9$ & $42.3 \pm 3.3$ & \\
\hline Intracranial volume $\left(\mathrm{cm}^{3}\right)^{\mathrm{d}}$ & $1495.0 \pm 144.6$ & $1524.5 \pm 147.8$ & $1495.5 \pm 154.3$ & $F(2,226)=0.56, p=0.575$ \\
\hline
\end{tabular}

Values represent means \pm SDs.

Post-hoc tests showed: ${ }^{a} p<0.001$, deeper than in both patient groups; ${ }^{b} p<0.001$, deeper than in schizophrenia; and ${ }^{c} p<0.001$, deeper than in left hemisphere.

${ }^{d}$ Analyzed using the ANCOVA, with age as a covariate, diagnosis and gender as between-subject factors. There was a significant gender difference [male $>$ female; $F(1,226)=136.23, p<0.001$ ] 


\section{Left hemisphere}

\section{Right hemisphere}
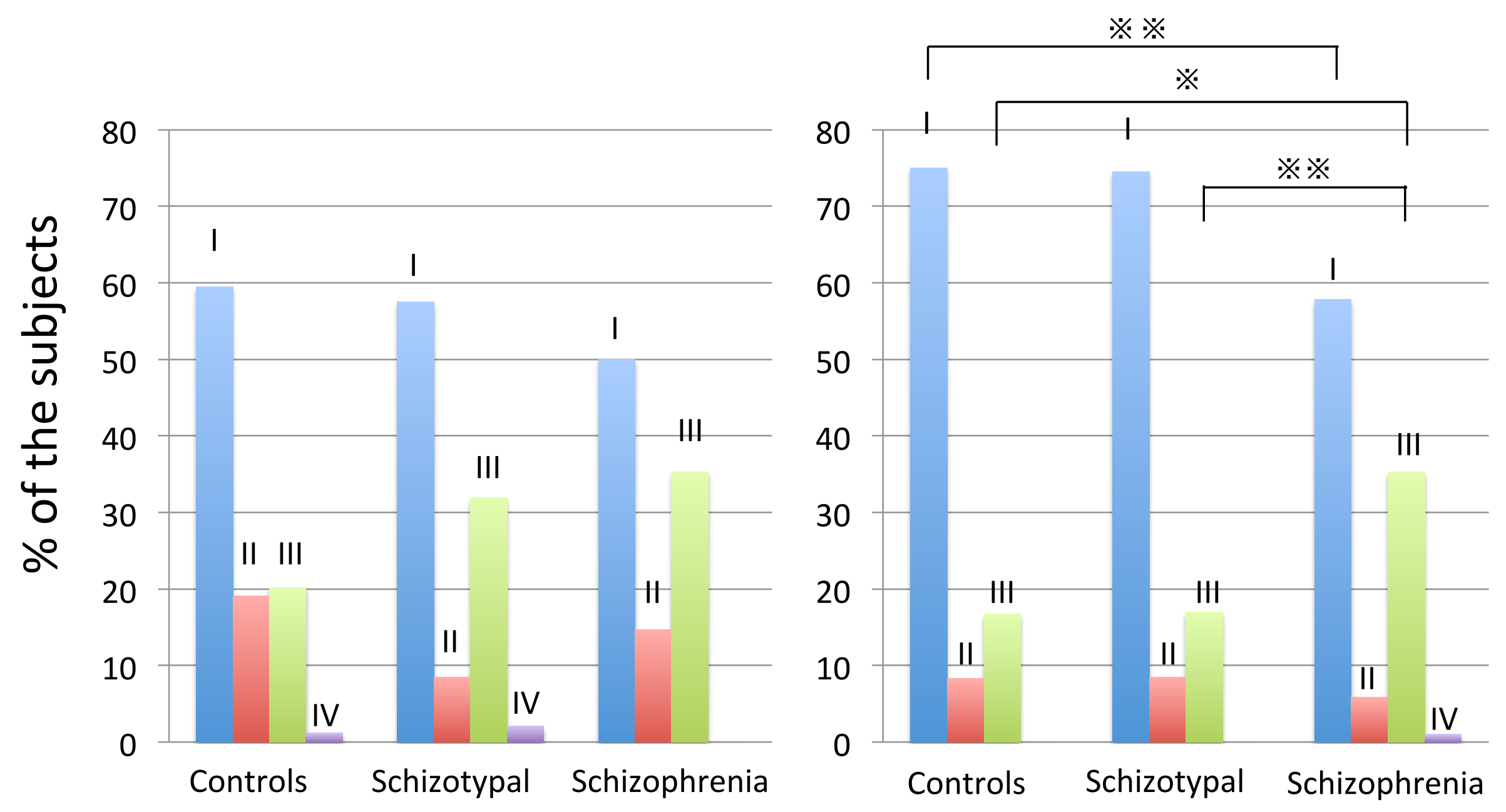